\title{
Dance.Draw: Exquisite Interaction
}

\author{
Celine Latulipe \\ Department of Software and Information Systems \\ University of North Carolina at Charlotte \\ Charlotte, NC, USA 28233 \\ +1704 687-8195 \\ clatulip@uncc.edu
}

\author{
Sybil Huskey \\ Department of Dance and Theatre \\ University of North Carolina at Charlotte \\ Charlotte, NC, USA 28233 \\ $+1704687-2503$ \\ sdhuskey@uncc.edu
}

\begin{abstract}
We present a light-weight, low-cost, portable system for interactive dance performances in which projected visualizations reflect the dancers' movements in real-time. This project has spawned a new and exciting collaboration between dance and technology at UNC Charlotte.
\end{abstract}

\section{Categories and Subject Descriptors}

H.5.2 [Information Interfaces and Presentation]: User Interfaces - evaluation/methodology, user-centered design.

\section{General Terms}

Design, Human Factors

\section{Keywords}

Performance interactions, dance, gyroscopic mouse, bimanual interaction, collaborative interaction.

\section{INTRODUCTION}

We have created a new interactive system for dance, called Dance.Draw. The Dance.Draw system allows dancers to control a projected visualization with their movements ( Figure 1). Rather than using cumbersome and expensive technologies such as motion capture suits or video tracking, the Dance.Draw system uses commodity devices: wireless USB mice. This makes the system portable, easy to setup, and inexpensive, while maintaining the ability to track motion and use that motion as spatial input for the projected visualizations.

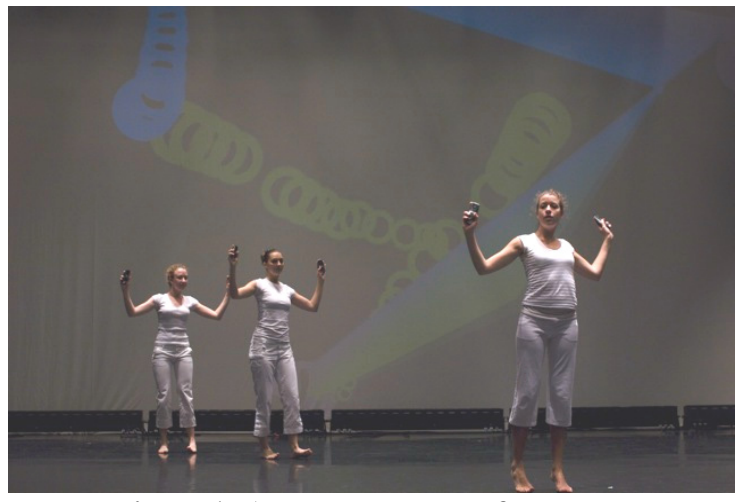

Figure 1: A Dance.Draw performance.

(c) The Author 2008.

Published by the British Computer Society
The Dance.Draw system is part of a larger ongoing project called Exquisite Interaction, in which the idea is to support multiple artists or performers, each using multiple input devices to work together in a shared space and to collaborate on the creation and control of visual artifacts. While it is possible that each artist or performer can each control their own object, the interaction becomes 'exquisite' when the artists work together to create or control the same object. The name exquisite interaction is derived from the parlor game 'exquisite corpse', in which one person draws half of a picture on half of a piece of paper, folds the paper and gives the blank side to another person to finish. The exquisite corpse game has been adapted as an exercise for artists, because it forces artists to be influenced by and react to one another in an immediate way. The idea of exquisite corpse has also been adapted to music, film, and animation.

Our research contribution is a light-weight, inexpensive, portable system for the production of interactive dance performances in which dancers can use the motion of their bodies in space as input to a projected visualization. In this paper we describe related work and then situate our system within that work. We describe three dance performances that were produced with the Dance.Draw system and talk about the possible directions for this research in the future.

\section{RELATED WORK}

Theories and practices in dance can be applied to creating more embodied interaction techniques, as described by Dourish [3], and others [7][8] in the Computer-Supported Collaborative Work (CSCW) and Human-Computer Interaction communities. However, applying technology to dance is the direction that this paper is concerned with. Much of the CSCW literature is focused on supporting task-based work. While dance is work for dancers, it is about creativity, movement and expression, rather than task completion. Therefore, we choose to focus in this short paper on situating Dance.Draw within the body of work on interactive forms of dance in which digital media play a role.

Technology and new media can play a role in dance education, dance experimentation, choreography and in dance production. It can also become the subject matter for dance [1]. Merce Cunningham's 'Biped' work, making use of the Life Forms/Dance Forms computer animation software was one of the first forays into interactive, technology-mediated dance [2]. This has been followed by numerous interactive dance systems, many of which have used vision-based motion-tracking of the dancers during performance, and then applying the data to the generation or transformation of music and visuals, as described in [10] and [13]. The Body-Brush system tracks a single performer with a vision-based system and uses the data to create artwork [5]. Some work has been done in augmented 
dance using full motion capture suits [10]. Other work has used sensors attached to the dancers to mediate with MIDI-based technology. And the work of James et al. is revolutionizing the ability of machines to interpret dancers' movements [6]. The EyeCon system has been used by Palindrome [http://www.palindrome.de] to capture video of dance performances and then process, augment and display those video clips as part of interactive dance performances. Finally, there is also research in mediating the creation of dance over the Internet [11][12].

The Dance.Draw system is a co-located system for the creation of interactive dances that are light-weight and require no vision equipment or processing. Dance.Draw builds on our previous work in symmetric bimanual interaction. Dance.Draw emerged out of a collaborative, two-handed drawing application called symDrawEI. symDrawEI allows multiple artists to draw on the same canvas with each artist controlling two cursors using a mouse in each hand. The system allows the artists to draw individually or to work together to create more complex shapes. Both styles of interaction fall under the category of symmetric interaction, where both of a person's hands work together at the same level of temporal and spatial detail (this is in contrast to asymmetric interaction, in which the dominant hand performs detail work while the non-dominant hand plays only a supporting role [4]). While there are many tasks that are obviously asymmetric (hand-writing is the canonical example), dance is an art form that uses the whole body and it clearly makes sense to apply a symmetric input paradigm to interactions based on dance movements.

\section{THE DANCE.DRAW SYSTEM}

\subsection{System}

The Dance.Draw system is a Mac application built in $\mathrm{C} / \mathrm{C}++$ using the Carbon and OpenGL libraries. The system takes input from multiple pointing devices and turns those spatial input streams into visualizations that are projected onto a wall or screen behind the dancers, in real time. The system outputs both the programmed visualization and a selected audio track. Dance.Draw will run on any Mac OSX system; for our current performances, the system has been running off of a MacBook Pro portable computer. We have used high resolution projectors (capable of projecting at $1024 \times 768$ or higher), but that may not be necessary, depending on the complexity of the visualizations created. All of the venues where we have held performances have been equipped with high-resolution projectors.

\subsection{Input}

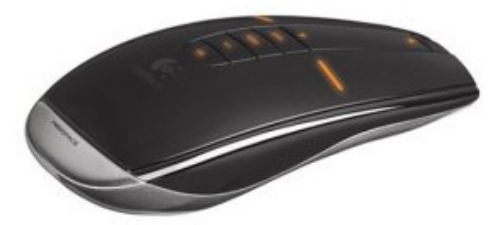

Figure 2: The Logitech MX Air Mouse.

The input devices that we are using are Logitech MX Air mice (see Figure 2), which are wireless, gyroscopic mice designed for controlling digital media centers in the home. Unlike regular optical mice, these gyroscopic mice do not have to be used on a surface, but can be used in mid-air (hence the name Air mouse). Because these devices are designed for use in the living room rather than in the office, they are more aesthetically pleasing than a typical mouse, and in fact are more aesthetically pleasing than the typical media center remote control.
The Logitech Air mice use wireless technology and although the devices are advertised with a 30-foot tracking range [http://www.logitech.com], we found that they actually tracked successfully up to 100 feet away from the USB wireless receivers, making them suitable for performances in both large and small venues.

\subsection{System Cost}

The cost of the Dance.Draw system in its current instantiation is approximately $\$ 1000$ ( $\$ 150$ per mouse for six mice, plus two USB hubs). A projector and a computer to run Dance.Draw are also required. We anticipate that most theater venues have digital data projectors, and the Dance.Draw application will run on any Mac OSX system.

While $\$ 1000$ in equipment is somewhat costly for a typical dance group, it is much cheaper than the equipment necessary for other interactive dance setups. In comparison, interactive dance systems with custom-built floor sensors, motion capture suits, etc. are much more expensive (for example, a ShapeWrap III basic system costs approximately US $\$ 32,000$ for a single motion capture suit [http://www.motion-capturesystem.com/shapewrap-basic.php]). Given this, we feel that our system offers a practical and inexpensive system for interactive dance.

\subsection{Input Grouping}

The current Dance.Draw system implementation assumes that each dancer will carry two mice (one per hand), and therefore groups the spatial input streams from the mice into pairs. This is the default setup, and in this configuration, the two spatial input streams from each dancer can be used to control a single object, or two separate objects. Thus, with three dancers, the default system configuration allows simple control of six individual objects or more complex control of three objects.

An alternate configuration can be activated with a keyboard press, where the six spatial input streams are grouped together to control a more complex visualization.

The USB system assigns IDs to the mice as they are plugged in, therefore the current system requires some initial setup to determine which mice are paired together, so that each dancer has a pair of mice that can control a single visual object. This can be done by either identifying each mouse with some type of visual tag and then controlling the order in which the mouse receivers are plugged into the computer, or by plugging them in randomly and running the system to determine how the system has paired the mice.

\subsection{Visualizations}

Our initial foray into interactive dance visualization has consisted of a short, structured improvisational dance performance with three phases of visualization. Because this system so far has been considered a technological 'proof of concept', the visualizations were programmed by a non-artist, and have been relatively unsophisticated. The benefits of a lowcost, portable system such as Dance.Draw are that it is easy to rapidly prototype and test out various visualizations and configurations, since this can be done anywhere without a lot of equipment setup. Because of this, we were able to easily vary the visualizations between the three performances, testing out different visual ideas.

The system starts off in the standard paired configuration, and in the first phase each dancer controls the translation of two objects, one with each hand. The second phase of the dance 
involves switching the system to the grouped mode and having the dancers collaboratively control a complex object with their movements. The third phase of the dance involves reverting to the paired mode and having three separate objects in the visualization, one controlled by each dancer. These visualizations will be described in more detail for each performance in the following section.

\section{INITIAL PERFORMANCES}

The first Dance.Draw performance was produced as part of a fund-raising kickoff event for the Arts and Science Council of Charlotte, a local umbrella organization for the arts. This event was attended by approximately 100 people from the campus and the Charlotte community and was held in a small classroom style auditorium. There was no formal stage for this performance, however the floor space in front of stadium seating was sufficient. In this venue, the visualization was projected onto a large screen hanging on the wall, the bottom edge of the screen was approximately 12' from the floor. Because of the room setup, the attention of the audience was divided: they could focus on the dancers or on the visualization, but they could not easily focus on both at the same time.

The music that the choreographer chose for the three performances described here was a piece called "Water Moon", by Andreas Vollenweider. The impressionistic quality and three-part structure of the piece provided a sound environment that resonated with the initial visualization images shown to the choreographer. The music also fit the time constraint of the first performance.

The performance was a structured improvisation in which the dancers came in, one at a time, and picked up a pair of mice each. The mice appeared sleek and dark in the dim room and were barely visible in the hands of the dancers. As soon as a pair of mice were picked up, a ring shape with slowing changing colors appeared on screen. The dancer's hand positions, through the mice, controlled opposite corners of the ring's invisible enclosing rectangle. So, for example, if the dancer's arms were spread wide, the ring would become large, and as the dancer moved around the stage the position of the ring would change. Once all three dancers were on stage, there were three rings in the visualization. At a specific crescendo in the music, a grouping function was applied (manually, by pressing a button on the keyboard), and the visualization changed from three rings to a single, seven-sided polygon, with each dancer controlling the position of two of the polygon's vertices. This dynamic polygon was nicknamed the flying origami visualization. It is this part of the dance that most exemplifies what we mean by exquisite interaction, because all of the dancers' movements affect the single object projected on screen. At a second crescendo, the grouping function was turned off and the dancers were once again controlling a third visualization of three separate objects. As the music came to an end, the dancers stopped dancing one at a time, setting down their mice. When the music finished, a random pattern was left on screen based on the final motions of the dancers.

The second performance was in a ballroom, where the dance was performed as part of an awards banquet. The visualizations changed somewhat for this performance: motion tracing was added, making each shape's recent motion path visible. The audience of 130 were seated at round tables, and the dancers performed in an open area in the middle of the room. The visualizations were projected synchronously on four screens along one side of the rectangular room. Thus, this venue also led to a divided attention issue for some audience members. Despite this, the response from the audience was very positive.
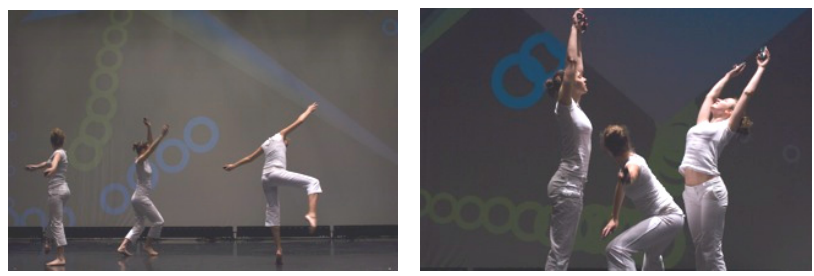

Figure 3: Photos of a Dance.Draw performance showing the motion traced rings and a part of the 'flying origami' visualization controlled by the dancers' movements.

The venue for the third performance of the Dance.Draw production was ideal. This production took place in a theatre, and was part of the program for the Visualization in the World Symposium at UNC Charlotte. There were approximately 150 people in the audience for this performance, and the visualization was projected onto the full wall at the back of the theatre stage. Thus, there was no divided attention problem for the audience: the dancers appeared super-imposed in front of the visualization. In this performance, the visualization changed again. In the first phase of the dance, the dancers each controlled two ring objects. The rings didn't change in size, but did have motion path traces. Thus, there were six objects on screen. Then, these ring traces remained during the flying origami phase of the dance, providing a more continuous narrative (Figure 3). Finally, the third phase consisted of three recursive, wavering, ring visualizations.

The variety of venues where performances were successfully staged attest to the portability and light-weight setup of the Dance.Draw system. Each performance required less than 10 minutes to setup the laptop, mice and projector hookup, plus some additional time to play with venue light levels and to adjust the choreography to the room/stage setup.

\subsection{Choreographer's Experience}

The ability to create shape and gesture with the body while also manipulating visual images in the background provided an interesting creative opportunity for the choreographer. The choreographer was initially concerned about the dancers dropping the mice. However, this only happened once in a rehearsal, and the dancers quickly adapted to holding the mice while dancing. Since the dancers were holding mice in each hand, movements initially tended to be arm-driven. Because hand support was not possible while holding the mice, the movement vocabulary generally stayed on mid-body to fullbody level. This level constraint was also partly an artifact of the first two performance venues, where the audience would have difficulty seeing floor-level movements.

The first choreographic consideration focused on the quality (time, space, energy) and range (spatial dimension) of movements in relationship to the resultant images. A challenge was to make the dance have its own choreographic integrity and not simply exist to manipulate the visualizations. The choreography was therefore designed to have the dance juxtapose with the visualizations in shape and motion with neither dominating the other.

\subsection{Dancers' Experiences}

The dancers involved in the project were students enrolled in the Dance program at UNC Charlotte. They had both positive and negative reactions to the Dance.Draw productions. They found the concept interesting and fun, but were occasionally 
distracted by wanting to watch the visualizations they were controlling. This was an issue because the visualizations were different in each performance, and the dancers hadn't necessarily had a chance to see the new visualizations prior to each performance. This issue will likely disappear when the system is used in a more substantial, rehearsed, production. The dancers enjoyed controlling the visualizations with their movements and liked that the visualizations were slightly different each time, even when the visualization code was unchanged.

The dancers did express some frustration at the limitation in movement caused by having to hold the mice. For example, the dancers were unable to do 'floor work' or poses that required hand support. While this can obviously be addressed by using a different tracking technology, the choreographer does not feel that we have fully explored the movement space with the micein-hand constraint, and that there are a variety of possible workarounds that could result in interesting new choreography.

\section{SUMMARY AND FUTURE WORK}

We have presented the Dance.Draw system for interactive dance performances using commodity devices. Three performances have been described to demonstrate the system's potential. Dance.Draw provides exciting opportunities and challenges for choreographers and dancers to engage with audiences using technology that goes far beyond the traditional lighting and sound effects. The system also allows programmers to prototype and easily test different visualizations with dancers because of the minimal equipment setup. While we have not yet tried to formally gather feedback from audiences, informal audience comments suggest that the performances have been very stimulating. We are interested in investigating ways to get feedback from the audience during the Dance.Draw performances, and possibly to incorporate that feedback into the visualization. To this end, we are looking at hedonic affective response measurements through the use of sliders or dials [4] and hope to apply this to a longer dance planned for production in Fall, 2008.

There are a huge variety of options for expanding this work. In terms of alternate input, we could use Nintendo Wiimotes as the input devices, but the Wiimotes do not have the same sleek aesthetic as the Air Mice. As an alternative, we may try using sensors instead of (or in addition to) the Air Mice, if we can do so without compromising the portability and affordability of the system. The current visualizations were designed by a computer scientist. Obviously, much more interesting and aesthetically pleasing visualizations could be created with the involvement of artists.

In summary, we are pleased with this application of twohanded symmetric interaction to creating a portable and inexpensive system for interactive dance, and we look forward to exploring this interdisciplinary space further.

\section{ACKNOWLEDGMENTS}

We would like to acknowledge Heather Freeman and Annabel Manning for participating in the initial exquisite interaction work. This work was supported by the Charlotte Visualization Center and the UNC Charlotte Department of Dance and Theatre.

Production Credits for the initial Dance.Draw Performances:

Dance.Draw System: Celine Latulipe

Visualizations: Celine Latulipe
Choreography: Sybil Huskey

Dancers:

Catherine Ciemier (first and third performances)

Sybil Huskey (second performance)

Megan Jones

Amanda Martin

\section{REFERENCES}

[1] Burg, J. and Luttringhaus, K. 2006. Entertaining with science, educating with dance. Computers in Entertainment. 4, 2 (Apr. 2006), 7.

[2] Calvert, T. 2007. Animating dance. In Proceedings of Graphics Interface 2007 (Montreal, Canada, May 28 - 30, 2007). GI '07, vol. 234. ACM, New York, NY, 1-2.

[3] Dourish, P. Where the Action Is: The Foundations of Embodied Interaction. MIT Press, 2001.

[4] Guiard, Y. Asymmetric division of labour in human skilled bimanual action: The kinematic chain as a model. Journal of Motor Behaviour, pages 486-517, 1987.

[5] Ip, H. H., Hay, Y., and Tang, A. C. 2002. Body-Brush: a body-driven interface for visual aesthetics. In Proceedings of the Tenth ACM international Conference on Multimedia (Juan-les-Pins, France, December 01 - 06, 2002). MULTIMEDIA '02. ACM, New York, NY, 664-665.

[6] James, J., Ingalls, T., Qian, G., Olsen, L., Whiteley, D., Wong, S., and Rikakis, T. Movement-based interactive dance performance. In Proceedings of the 14th Annual ACM international Conference on Multimedia (Santa Barbara, CA, USA, October 23 - 27, 2006). MULTIMEDIA '06. ACM, New York, NY, 2006, 470480.

[7] Kjölberg, J. 2004. Designing full body movement interaction using modern dance as a starting point. In Proceedings of the 5th Conference on Designing Interactive Systems: Processes, Practices, Methods, and Techniques (Cambridge, MA, USA, August 01 - 04, 2004). DIS '04. ACM, New York, NY, 353-356.

[8] Levisohn, A. The Body as a Medium: Reassessing the Role of Kinesthetic Awareness in Interactive Applications. In Proceedings of the $15^{\text {th }}$ Annual ACM International Conference on Multimedia (Augsberg, Bavaria, Germany, September 23 - 28, 2007). MULTIMEDIA'07. ACM, New York, NY, 2007, 485-488.

[9] Lottridge, D. Hedonic Affective Response as a Measure of Human Performance. Technical Report, Interactive Media Lab, University of Toronto, 2007.

[10] Meador, W. S., Rogers, T. J., O'Neal, K., Kurt, E., and Cunningham, C. 2004. Mixing dance realities: collaborative development of live-motion capture in a performing arts environment. Computers in Entertainment. 2, 2 (Apr. 2004), 12-12.

[11] Popat, S., Ng, K., and Smith-Autard, J. 2000. Hands-on dance project: creative dance collaborations online. In Proceedings of the Third international Conference on Collaborative Virtual Environments (San Francisco, California, United States). E. Churchill and M. Reddy, Eds. CVE '00. ACM, New York, NY, 203-204.

[12] Sheppard, R., Wu, W., Yang, Z., Nahrstedt, K., Wymore, L., Kurillo, G., Bajcsy, R., and Mezur, K. 2007. New digital options in geographically distributed dance collaborations with TEEVE: tele-immersive environments for everybody. In Proceedings of the 15th international 
Conference on Multimedia (Augsburg, Germany, September 25 - 29, 2007). MULTIMEDIA '07. ACM, New York, NY, 1085-1086.
[13] Wechsler, R. and Weiss, F. Motion Sensing for Interactive Dance. IEEE-Pervasive Computing, Mobile and Ubiquitous Systems, Jan-March 2004. 